Diskurse ballen können - beispielsweise in Form von sozialen Bewegungen (Pross \& Klewes, 1984, S. 7-9). Die «Masse» kann daher als Bedingung für die Ausformierung von Teil- oder Gegenöffentlichkeiten betrachtet werden. Demokratische Spannungen entstehen vor allem durch die Kritik dieser Teilöffentlichkeiten an Eliten, die in der klassischen Soziologie vorwiegend positiv konnotiert werden und als Gegenpol zur affektiven Masse stehen (Hartmann, 2004). Interessant ist vor allem die dichotome Unterscheidung zwischen diesen beiden Polen, wie sie sich auch in Öffentlichkeits- und Gegenöffentlichkeitskonzepten wiederfinden. Ich gehe davon aus, dass diese dichotome Betrachtung vor allem in digitalen Öffentlichkeiten nicht tragbar ist und dem Phänomen der Gegenöffentlichkeiten im digitalen Raum mehr Aufmerksamkeit geschenkt werden muss, wie im folgenden Kapitel erläutert wird.

\title{
V.2 Zum Stellenwert von Gegenöffentlichkeiten in digitalen Gesellschaften
}

Die Metapher der Longtail-Öffentlichkeit (z. B. Eisenegger, 2021) in digitalen Sphären zeigt deutlich, dass eine Unterscheidung in unterschiedliche Öffentlichkeitsebenen, wie beispielsweise von Gerhards und Neidhardt (1991) antizipiert, in dieser Form nicht mehr denkbar ist. Vielmehr konstituiert sich ein Sammelsurium an Akteur_innen, das im digitalen Raum öffentlich kommuniziert - vor allem aufgrund der partizipativen Möglichkeiten, die Digitalplattformen bieten. Viele Autor_innen, die sich mit Gegenöffentlichkeiten theoretisch und empirisch auseinandersetzen, erachten den digitalen Wandel aufgrund von Globalisierungstendenzen, Vernetzungsmöglichkeiten oder partizipativem (Bürger_innen-)Journalismus als massgeblich für deren Entwicklung (z. B. Engesser \& Wimmer, 2009; Fenton \& Downey, 2003; Kaiser \& Rauchfleisch, 2019; Pfetsch et al., 2018; Schink, 2020). Die Konnektivität des Internets als Basis für die Etablierung von Gegenöffentlichkeiten brachte zudem auch aus einer methodisch-empirischen Perspektive neue Möglichkeiten für die Erforschung digitaler Öffentlichkeiten. Diesbezüglich werden vor allem Digitalplattformen und dort existierende Netzwerkstrukturen untersucht, meist basierend auf Daten, die von Digitalplattformen selbst zur Verfügung gestellt werden. Die inhaltliche Perspektive der Netzwerkstrukturen, das Dazwischenliegende, nimmt dabei häufig einen geringeren Stellenwert ein, obwohl dies wesentlich erscheint, beispielsweise um Fragmentierungstendenzen («Echokammern» oder «Filterblasen») insbesondere bezüglich öffentlicher und gegenöffentlicher Gruppen interpretieren zu können. Die vorliegende Arbeit setzt sich daher stärker mit Relationen als mit Strukturen in Netzwerken auseinander, basierend auf theoretischen Grundlagen der relationalen Soziologie (Emirbayer, 1997), 
die davon ausgeht, dass Narrative zwischen Akteur_innen wesentlich für die Beschreibung von Beziehungen sind.

Als konkretes Phänomen werden in der vorliegenden Arbeit alternative Nachrichtenmedien oder Alternativmedien als Beispiel für Gegenöffentlichkeiten untersucht. Obwohl alternative Medien historisch betrachtet eine lange Tradition aufweisen, z. B. in Form der Meinungspresse im Zuge der Französischen Revolution (Habermas, 2018 [1962]) oder der Alternativpresse der neuen sozialen Bewegungen (Wimmer, 2015), scheinen sie in digitalen Öffentlichkeiten eine neue Bedeutung oder Rahmung erlangt zu haben. Als Opposition gegenüber der hegemonialen politischen und medialen Öffentlichkeit (z. B. Holt, 2020; Holt et al., 2019) treten sie als Kritik- und Kontrollinstanz gegenüber dem «Mainstream» auf und stehen in diesem Sinne relational zur Öffentlichkeit. Wie aus dem Forschungsstand ersichtlich wurde, besteht trotz einschlägiger Studien durchaus Forschungsbedarf hinsichtlich des Themas «Alternativmedien». Erstens mangelt es an einer aktuellen Begriffsdefinition, die dem digitalen Wandel der öffentlichen Kommunikation gerecht wird (vgl. hierzu auch Holtz-Bacha, 2015). Zweitens richtet sich der aktuelle Fokus in der (theoretischen wie auch empirischen) Literatur vorwiegend auf den US-amerikanischen Raum und rechtspopulistische, desinformative oder verschwörungstheoretische Medien (z. B. Figenschou \& Ihlebæk, 2018; Haller \& Holt, 2018; Haller et al., 2019; Hollander, 2018; Holt et al., 2019; Zannettou et al., 2017). Dem deutschsprachigen Raum und seinen unterschiedlichen Angeboten an Alternativmedien im Internet wird indes zu wenig Beachtung geschenkt. Vor allem in pluralen Mediensystemen ist anzunehmen, dass ein Fokus auf alternative Medien, die antidemokratisch agieren und desinformative Inhalte verbreiten, $\mathrm{zu}$ einschränkend ist - insbesondere, wenn die historische, demokratische Perspektive alternativer Medien berücksichtigt wird.

Mithilfe eines explorativen, multi-methodischen Forschungsdesigns soll diese Lücke geschlossen werden. Mit einem Fokus auf alternative Online-Nachrichtenmedien - im Sinne deutschsprachiger Websites, die aktuelle Nachrichten verbreiten und nicht $\mathrm{zu}$ den etablierten Informationsmedien zählen - soll untersucht werden, welche Formen alternativer Nachrichtenmedien in Deutschland, Österreich und der deutschsprachigen Schweiz aktuell existieren, anhand welcher Kriterien diese definiert werden können und inwiefern diese auf Social-Media-Plattformen (am Beispiel Twitter) untereinander und mit der medialen und politischen Öffentlichkeit vernetzt sind. Nachfolgend werden die konkreten Forschungsfragen formuliert. 